\title{
Infrapatellar Branch of the Saphenous Nerve Injury: Implications for the Physical Therapist
}

Irwin S. Thompson, MPT, EdD

Department of Physical Therapy, California State University, Sacramento 6000 J St., Sacramento, CA 95819, United States.

Article Details

Article Type: Special issue

Received date: $03^{\text {rd }}$ June, 2021

Accepted date: $29^{\text {th }}$ June, 2021

Published date: $01^{\text {st }}$ July, 2021

"Corresponding Author: Irwin S. Thompson, MPT, EdD, Department of Physical Therapy, California State University, Sacramento 6000 J St., Sacramento, CA 95819, United States. E-mail: irwin.thompson@csus.edu

Citation: Thompson, I.S. (2021). Infrapatellar Branch of the Saphenous Nerve Injury: Implications for the Physical Therapist. J Rehab Pract Res, 2(2):120. https://doi.org/10.33790/jrpr1100120

Copyright: ( 2021 , This is an open-access article distributed under the terms of the Creative Commons Attribution License 4.0, which permits unrestricted use, distribution, and reproduction in any medium, provided the original author and source are credited.

\section{Abstracts}

Injury to the infrapatellar branch of the saphenous nerve (IBSN) is an underreported condition which has important implications for the physical therapist. Due to its location, the IBSN is especially vulnerable to damage following surgery, but it may also be injured through trauma or unknown causes. Patients with suspected IBSN injury may present with a variety of symptoms, including pain along the nerve distribution, paresthesia, hypoesthesia, anesthesia, and impaired functional activities and mobility. As the presence of IBSN injury may not be readily apparent, it is incumbent upon the physical therapist to recognize the signs and symptoms associated with this condition, and to administer a detailed clinical examination. Clinical evaluation can be difficult, as IBSN injury mimics many other conditions of the knee. Additionally, saphenous nerve anatomy exhibits wide variance, even within individuals, making diagnosis challenging. Upon suspicion of IBSN injury, the physical therapist should refer the patient for a comprehensive medical exam, which may include nerve blocks, nerve conduction studies, MRI, CT scan, or exploratory surgery. Most patients respond well to surgical intervention, including neurolysis or neurectomy, though conservative treatment options exist. These include nerve blocks or pharmacologic interventions. Physical therapy treatment is not well reported and may only be appropriate for specific etiologies of injury. Key words: saphenous nerve; infrapatellar branch; entrapment; injury; iatrogenic; physical therapy
\end{abstract}

\section{Introduction}

Injury to the infrapatellar branch of the saphenous nerve (IBSN) is an underreported condition $[1,2]$ which has important implications for the physical therapist. Due to its location, the IBSN is especially vulnerable to damage following surgery, [3-11] but it may also be injured through trauma or unknown causes [1,7,12-22]. In most individuals, injury may elicit minor symptoms, such as hypoesthesia or anesthesia [3]. However, in some, injury to the nerve can lead to debilitating pain $[1,9,10,13,23,24]$. Problematic for diagnosis, IBSN injury can mimic other conditions and may be overlooked $[6,10,25]$. Moreover, symptoms may not immediately manifest themselves following injury, leading to a confusing patient presentation [1]. It is, therefore, incumbent upon the physical therapist to recognize the hallmark characteristics of this condition and to undertake appropriate evaluation, treatment, and referral, as indicated. Unfortunately, reports of IBSN injury in the physical therapy literature are notably sparse.

This article will discuss the prevalence and etiology of injury to the IBSN, its anatomy, suggestions for clinical diagnosis, treatment, and implications for the physical therapist.

\section{Methods}

A literature review was conducted using PubMed, CINAHL and Google Scholar. Key terms included: saphenous nerve entrapment, saphenous nerve injury, saphenous nerve physical therapy, infrapatellar branch of saphenous nerve entrapment, infrapatellar branch of saphenous nerve injury, and infrapatellar branch of saphenous nerve physical therapy. Excluded sources included those prior to 1960 (except for historical reference), those in non-peer reviewed journals or texts, and those not available in English.

\section{Prevalence}

Injury to the saphenous nerve is not widely reported [1, 2], so its prevalence may be disputed. Determining actual prevalence is problematic, as the literature includes mostly case reports. No systematic review of IBSN injury is yet published. Notably, only one report of IBSN injury is found in the physical therapy literature [18].

Various authors disagree on the extent to which IBSN injury occurs. Mumenthaler [26] suggests that the saphenous nerve is complicit in less than $1 \%$ of the population who present with lower extremity pain. Notwithstanding these findings, reports of IBSN injury following knee surgery are high $[3,4,5,7,8,9,27,28]$. Abbott and Carpenter [29], as far back as 1945, highlighted the need to protect cutaneous nerves during surgery due to their susceptibility to injury during anterior and medial knee incisions. Nonetheless, iatrogenic nerve injury remains prevalent. Figueroa, et al. [3] reported that up to $77 \%$ of patients following knee arthroscopic procedures experienced sensory changes along the IBSN distribution. The authors further reported that electrophysiologic testing revealed IBSN injury in $68 \%$ of postsurgical knees. It has been suggested that $27.6 \%$ to $64.7 \%$ of anterior cruciate ligament (ACL) reconstructions, via either bone-patellar tendon-bone autograft or hamstring autograft technique, contribute to IBSN injury [4,7,28,30-36].

Despite reports of high prevalence, most patients may experience 
only minor sensory changes - such as hypoesthesia or anesthesia $[10,13]$ - in the nerve distribution that do not significantly impair daily function [3] Nonetheless, IBSN injury can produce severe pain and contribute to impairments with movement and mobility $[1,2,6,10,24]$.

\section{Anatomy}

The saphenous nerve - the longest branch of the femoral nerve - is purely sensory. It arises from the L3 \& L4 nerve roots [10,24], and emerges from the femoral nerve a few centimeters inferior to the inguinal ligament where it runs deep to the sartorius muscle and anterior to the femoral artery [24]. It passes lateral to medial within the fascia of the adductor canal [24], also known as Hunter's canal. This canal is a fascial channel found in the mid-thigh, extending from the femoral triangle proximally to the adductor hiatus distally [24]. The adductor hiatus is a tendonous opening in the adductor magnus, which allows for transmission of femoral vessels, including the saphenous nerve [24]. The borders of the adductor canal include the vastus medialis, the adductor hiatus, and the adductor magnus [24]. As the saphenous nerve emerges from the adductor canal it becomes more superficial and can be directly palpated [2.37.38]. The location where it emerges is not well-defined [24]. Lumsden and Kalenak [37] reported a mean distance from the superior patellar pole of $9.8 \mathrm{~cm}(7.5 \mathrm{~cm}-14.3 \mathrm{~cm})$, whereas Worth [2] described a mean distance of $10 \mathrm{~cm}$ from the medial femoral epicondyle, and Patterson [38] reported a mean distance of $10.5 \mathrm{~cm}(7.4 \mathrm{~cm}-14.1 \mathrm{~cm})$ from this same landmark. Once the saphenous nerve emerges from the adductor canal fascia, it is transmitted between the sartorius and gracilis tendons [38] where it splits into two branches: the sartorial branch, which extends along the medial leg; and the infrapatellar branch, which runs inferiorly and laterally to innervate the skin on the medial and anterior aspects of the proximal tibia and joint space $[10,24,38]$. Some authors have noted variations in which branching occurs within the adductor canal itself $[10,24,38]$. The infrapatellar branch will be explored more thoroughly due to its clinical relevance.

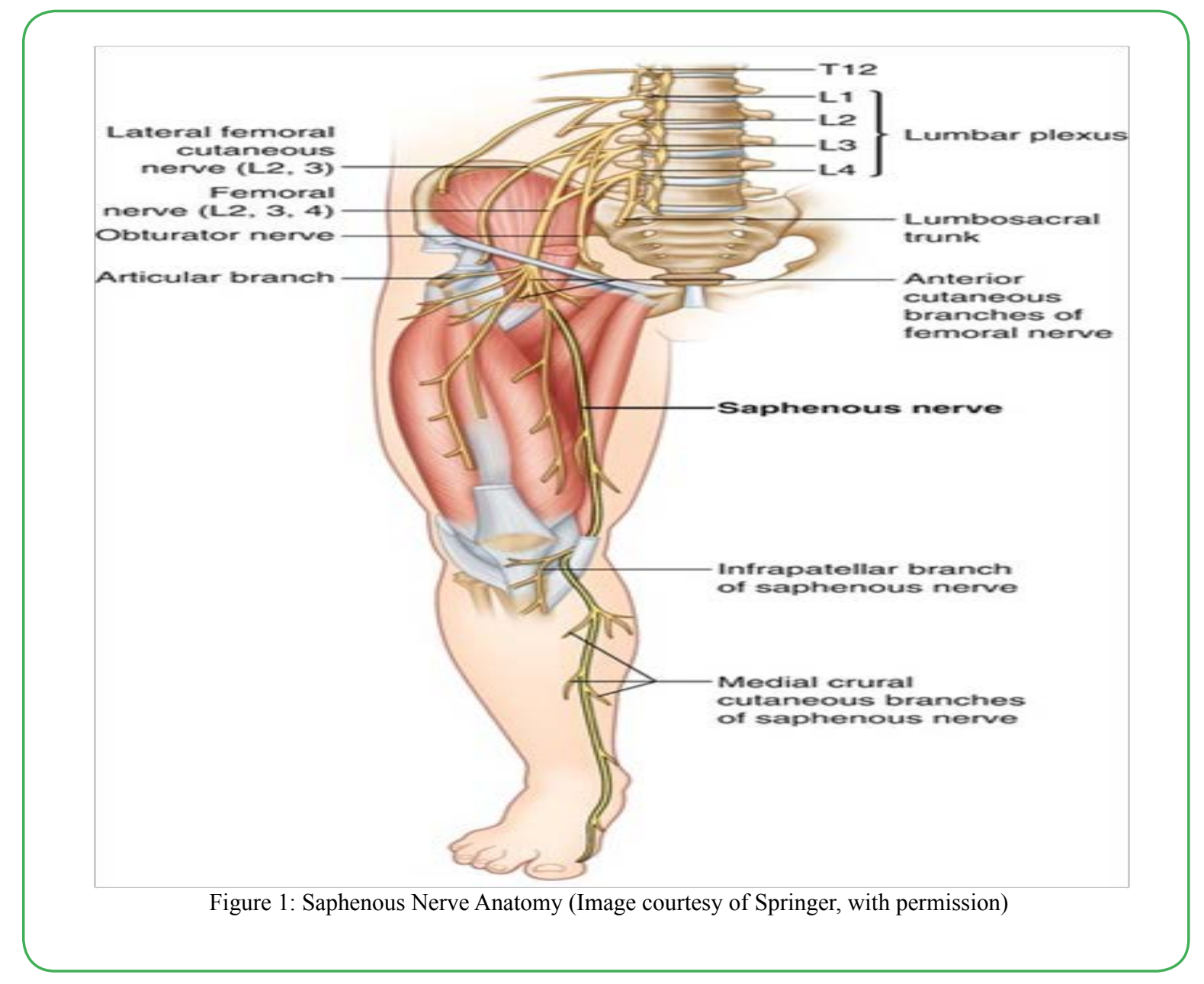

Locating the courses of each branch can be difficult, as anatomical variance exists between and within individuals. Notably, Arthornthurasook and Gaew-Im found only 50\% of examined specimens had identical anatomy, with variance in number and location of branches [40]. The presence of between one to four branches of the IBSN is widely reported [5,38-41]. Kartus, Eierhed, and Eriksson [39] identified one branch in $25 \%$ of specimens dissected, two branches in $63 \%$ of specimens, three branches in $10 \%$ of specimens, and four branches in 1.5 percent of specimens. The location of where each division emerges also varies, with identified branches located superficial to the sartorius on the medial knee $(62 \%)$, penetrating the sartorius $(22 \%)$, running parallel with the sartorius (14\%), and traveling posterior to the sartorius (3\%) [40].
Additionally, some branches arise in rare instances, not from the saphenous nerve, but from the femoral or obturator nerve [41].

Once divaricated from the sartorial division, the IBSN and its associated branches course inferiorly and laterally across the anterior aspect of the knee [43], with most branches passing the joint line medial to the patellar tendon [5]. Kartus, Eierhed, and Eriksson [39] found $98.5 \%$ of branches passing between the apex of the patella and the tibial tubercle with the remaining branches passing inferiorly to the tibial tubercle. Tifford [9] described the location of a superior and inferior nerve, in relation to a point $2 \mathrm{~cm}$ medial to the patellar ligament. The superior nerve could be located $5.1 \mathrm{~mm}$ superior to this point with the knee extended, and the inferior nerve could be located $34.6 \mathrm{~mm}$ inferior to this point. Alternately, Gali [42] located the primary nerve 
branch $2.61 \mathrm{~cm}$ inferior to the medial joint line along a line contiguous with the medial collateral ligament. Consequent to these potentially wide variations, the clinician must possess a detailed understanding of possible IBSN anatomy when conducting an examination.

\section{Etiology}

Iatrogenic causes are most commonly associated with IBSN injury. Most frequently, surgeries of the knee, such as ACL reconstruction with hamstring or patellar tendon grafting $[3,4,21,27,38,43]$, total knee arthroplasty (TKA) [10,21,23,43-45], and knee arthroscopy $[2,21,38,46]$ have been found to cause injury. Hamstring graft harvesting associated with ACL reconstruction appears particularly troublesome, as incisions for harvesting the semitendinosis tendon must pass close to the where the IBSN emerges between the sartorius and gracilis tendons $[4,42,47]$ Chambers $[48]$ has cautioned surgeons to pay careful attention with medial knee incisions due to the neuroanatomy of the region, though Kerver, et al. [49] concede no safe zone truly exists for portal placement. The direction of incision appears to play a role in the incidence of nerve injury, with a reported $64.7 \%$ injury rate following vertical incisions, $50 \%$ following horizontal incisions, and $27.6 \%$ following oblique incisions $[4,27]$ Less commonly, patients may suffer injury to the IBSN following saphenous vein harvesting during coronary artery bypass graft surgery [50-53].

Two sequalae of injury are described postoperatively: entrapment [38] and neuroma development [44,45,54,55,56,57]. Entrapment may occur due to misplaced sutures or post-operative scarring. Neuromas typically develop following transection of the nerve and are more common in pre-patellar medial knee incisions [45]. Subsequent to nerve transection, axonal regrowth is blocked, and terminal sprouts turn toward the cell body to create a painful tangle of nerve endings [56].

Non-surgical causes of IBSN injury are less prevalent. Macrotrauma from a fall $[6,18]$ or a direct blow to the medial thigh or knee $[6,15,21,22]$ have been reported. Additionally, overuse injuries from running $[12,17,20]$ have been suspected of causing damage to the IBSN. Spontaneous injury to the nerve, though less commonly suggested, may occur $[1,13,14,16,19,59,60]$.

\section{Clinical Presentation}

Patients seeking treatment with suspected IBSN injury may present with a variety of symptoms, though the most typical involve sensory changes along the nerve distribution in the medial and anterior surfaces of the knee. Most problematic of these sensory changes is pain $[1,10,23]$, which can be severe and debilitating [10]. Painful symptoms have been described as sharp, burning, and aching [23], as well as stabbing and a "fullness" in the knee [6]. Additional sensory changes may include hypoesthesia, anesthesia, paresthesia, allodynia and dysesthesia $[1,4,10,13]$. Sensory changes are always lateral to the point of injury or entrapment [9] as those align with the course of the nerve. Symptoms may be vague and non-localized, which presents diagnostic challenges $[1,10,23]$.

Patients with IBSN injury may complain of pain with active movement, such as walking, standing, negotiating stairs, or arising from a chair $[1,2,6,10,24]$. Specifically, contraction of the quadriceps [2], sartorius [13], hip flexor and adductor [18] muscles may elicit symptoms. Some patients have experienced symptoms at rest [1,24,54].

\section{Clinical Diagnosis}

The physical therapist may be the first practitioner to encounter the patient with signs and symptoms of IBSN injury, so suspicion of this condition warrants a thorough clinical examination. Though a differential diagnosis algorithm is not yet established, other similarly presenting pathologies should be ruled out. These include lumbar radiculopathy, sartorius tendonitis, pes anserine bursitis, medial collateral ligament injury, medial meniscus injuries, suprapatellar plica, osteochondritis dissecans, reflex sympathetic dystrophy (RSD), and deep vein thrombosis (DVT) [6,10,25].

The first component of an examination for IBSN injury involves a comprehensive medical and subjective history [10]. As most IBSN injuries are a result of surgical or mechanical trauma, a history of either of these events may be significant. The duration of the pain may be indicative of IBSN injury. Several reports document patients with recalcitrant symptoms that had eluded initial diagnosis and treatment and had been present for months to years [10,18,27]. Pain location must be ascertained and should be compared against known IBSN sensory distributions $[10,19,60]$. The history should include a description of the quality of the pain, as neurogenic pain exhibits unique characteristics that may be differentiated from other sources $[10,17]$. Activities that exacerbate or alleviate the pain may provide insight into the nature of the patient's symptoms. Those activities that require contraction of the quadriceps, sartorius, hip flexors and adductors, or that place stretch on the nerve, may be noteworthy $[2,13,18,19]$.

Observational assessment includes inspection for integumentary changes and should include a skin temperature assessment, as RSD has been associated with some IBSN injuries [10,49]. Should the patient present post-operatively, the physical therapist must carefully examine incision sites, particularly those around the medial knee. As mentioned, vertical incisions have correlated more highly with IBSN injury than horizontal or oblique incisions [4]. A history of trauma to the medial thigh or knee warrants examination for edema or hematoma in the regions of potential nerve injury.

A motor assessment involves muscle strength testing and deep tendon reflex testing. IBSN injury will not affect motor function, as the nerve is purely sensory, so the presence of motor loss may indicate alternate pathologies. However, muscle testing may elicit symptoms, particularly with testing of muscles previously noted as complicit to this pathology [2.13.18.19].

Vascular assessment should rule out arterial or venous compromise. Specifically, it may include palpation and assessment of the femoral and pedal pulses, as well as assessment of signs of DVT [10].

Sensory assessment includes palpation and Tinel's tests at key locations; specifically, at the adductor canal and along the medial aspect of the knee by the sartorius tendon proximal to its attachment at the pes anserinus $[10,16,19,20,44,45]$. These locations describe the transmittance of the primary branch of the saphenous nerve and the IBSN, respectively. Additionally, palpation along the known distribution of the IBSN may be informative, and should include, at a minimum, the skin over medial joint line, the medial tibial condyle, and the tibial tubercle $[1,10,23]$. Von Frey filament testing can also be utilized to assess sensory integrity along the IBSN distribution [10]. During sensory testing, the clinician is advised to be aware of the anatomical variance of both the main branch of the saphenous nerve and the potentially many branches of the infrapatellar division. As such, relying solely on textbook descriptions of the nerve's distribution may be inadequate. Instead, careful exploration of the region, including anatomical variances reported in the literature, is warranted. Figures 2 and 3 highlight the regions along the medial thigh and knee in which the saphenous nerve and its associated branches may be directly palpated. 


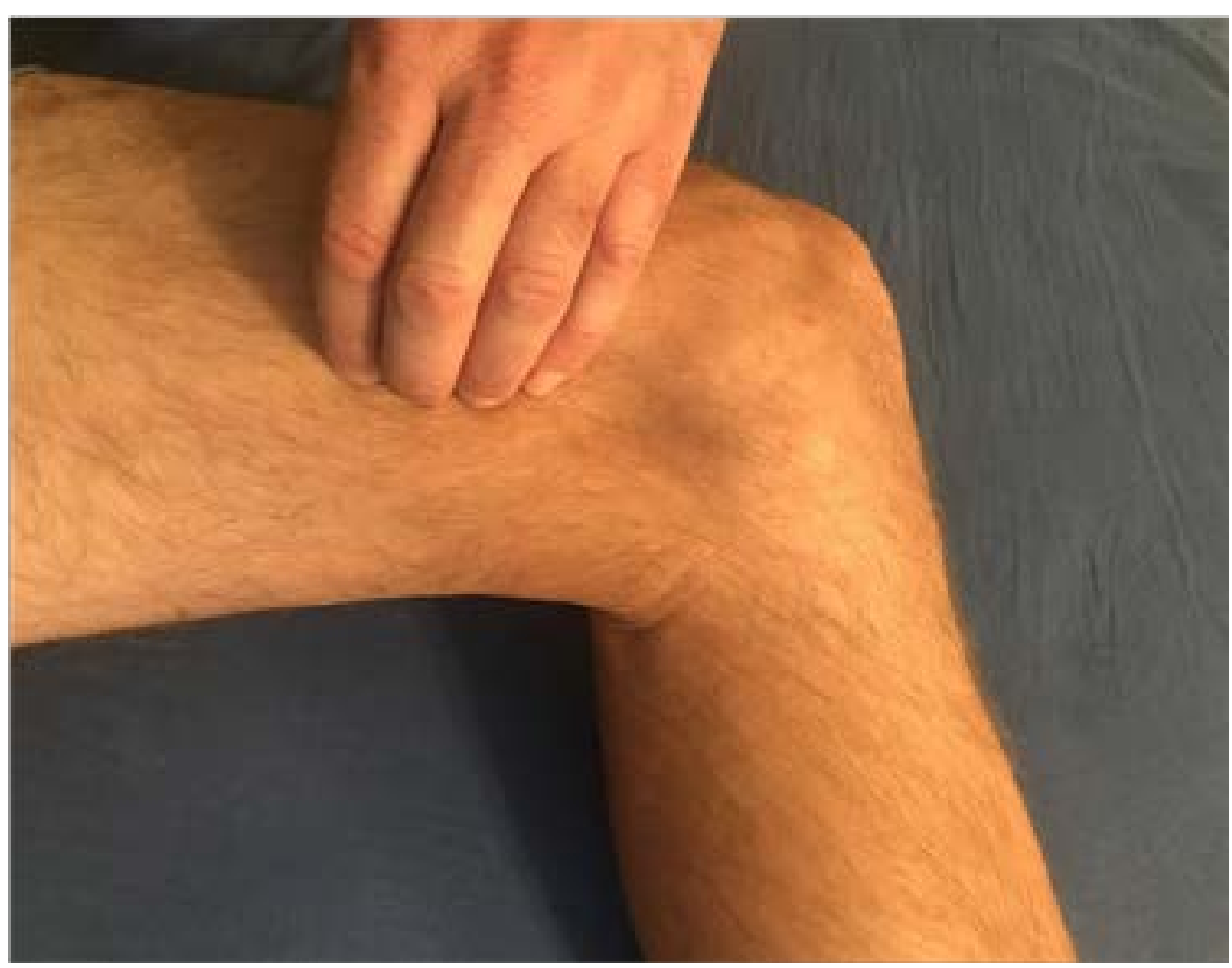

Figure 2: Palpation of the saphenous nerve at the adductor canal

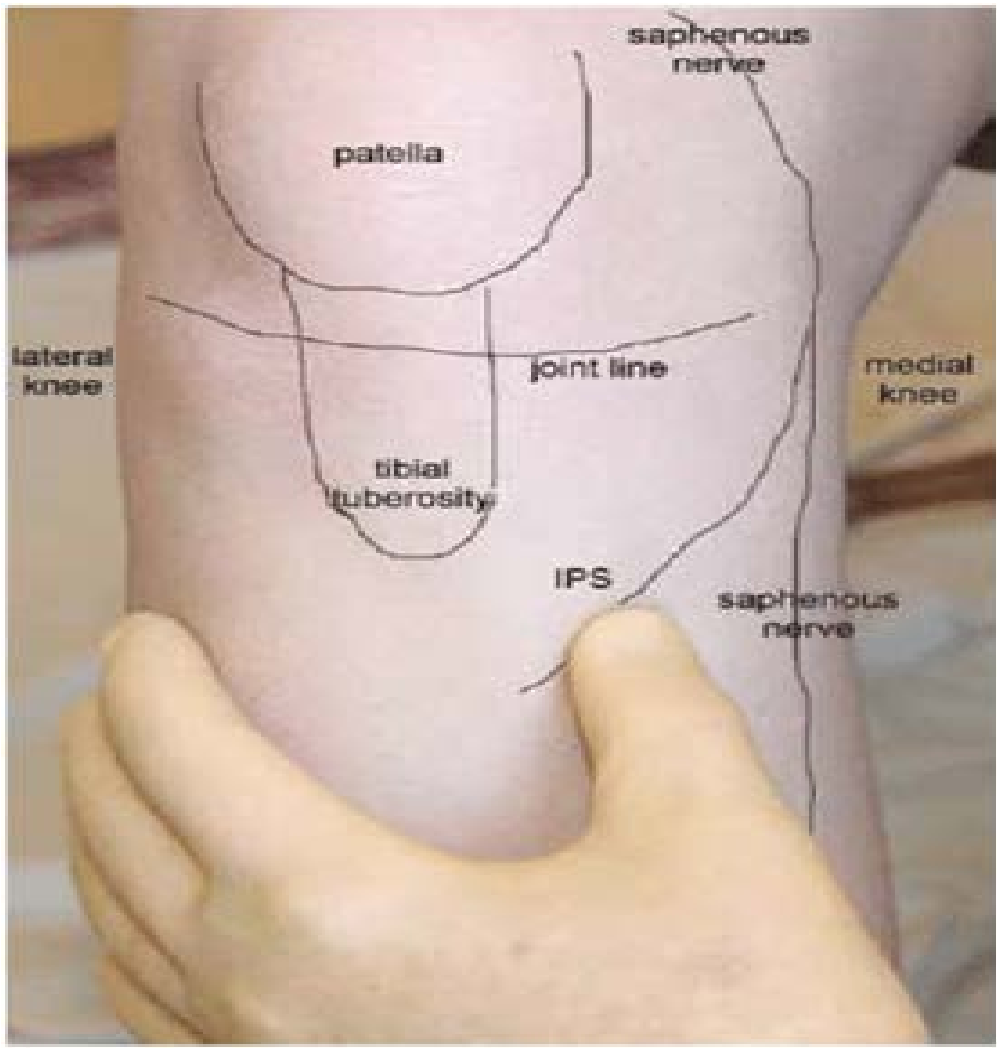

Figure 3: Palpation of the IBSN along the anteromedial knee (Image courtesy of Springer, with permission) 
Nerve tension testing may be incorporated as part of a comprehensive evaluation. The reverse Laségue's test (or prone knee bend test) has been documented for its utility in assessing both femoral nerve of which the saphenous nerve is a branch - and lumbar pathology
$[6,18,61]$. A positive test is unable to discriminate between peripheral and spinal nerve involvement; however, it may reveal a neurogenic condition. The clinician is advised to proceed cautiously with this test, as tension placed upon the nerve may exacerbate symptoms [19].

\begin{tabular}{|c|c|}
\hline Examination & Relevant Findings \\
\hline Subjective history & $\begin{array}{l}\text { History of knee surgery or medial thigh and knee } \\
\text { trauma } \\
\text { Recalcitrant symptoms lasting months to years } \\
\text { Neurogenic symptoms along the IBSN distribution } \\
\text { Pain with active movement that involves the } \\
\text { quadriceps and/or the sartorius muscles }\end{array}$ \\
\hline Observation/Integumentary & $\begin{array}{l}\text { Presence of incision sites (vertical }>\text { horizontal }> \\
\text { oblique) along medial knee } \\
\text { Integumentary and/or temperature changes } \\
\text { associated with RSD }\end{array}$ \\
\hline Motor assessment & $\begin{array}{l}\text { No predicted MMT or DTR change with IBSN } \\
\text { injury } \\
\text { Contraction of quadriceps, sartorius, hip flexors, } \\
\text { and hip adductors may elicit symptoms }\end{array}$ \\
\hline Vascular assessment & $\begin{array}{l}\text { Femoral and pedal pulses are not typically affected } \\
\text { with IBSN injury } \\
\text { Rule out presence of DVT }\end{array}$ \\
\hline Sensory assessment & $\begin{array}{l}\text { Pain, hypoesthesia, anesthesia, paresthesia, } \\
\text { allodynia and/or dysesthesia along nerve } \\
\text { distribution }\end{array}$ \\
\hline Nerve tension test & + Reverse Laségue's test \\
\hline Other tests \& measures & $\begin{array}{l}\text { Rule out involvement of associated soft tissue } \\
\text { structures, including the sartorius tendon, MCL, } \\
\text { meniscus, and pes anserinus bursa }\end{array}$ \\
\hline
\end{tabular}

If IBSN injury is suspected following a comprehensive examination, the physical therapist should refer the patient for further testing to confirm or refute the initial diagnosis. Medical diagnostic testing takes many forms. The current gold standard involves nerve blocks with Bupivacaine or Lidocaine [2,10,11,19,54,55,61]. Patients often report complete but temporary resolution of symptoms following nerve block [2,10,11,19,54,55,61]. Nerve conduction studies - either sensory nerve action potentials or somatosensory evoked potentials - may also be a part of an evaluation of suspected IBSN injury $[10,12,62-64]$. Some authors contest the reliability of these methods $[2,13,14,24]$, and it has been suggested that missed diagnoses may be consequent to focus on the primary branch of the saphenous nerve and not the infrapatellar branch [10]. Imaging, via magnetic resonance imaging (MRI) or computerized tomography (CT), is also utilized for diagnosis $[18,22,24,25,61,62]$. Occasionally, exploratory surgery is required to identify injury to the IBSN $[1,13]$.

\section{Treatment}

Treatment approaches depend upon the etiology of injury. As most reported cases of IBSN involve neuroma growth following surgical transection or entrapment due to perioperative scarring or suturing, additional surgical intervention is often indicated. Neurolysis or neurectomy are most widely reported $[2,13,22,31,15,16,62]$. Less common surgical approaches involve cryoablation [10] and radio frequency ablation [58]. Reported outcomes for these surgical interventions are overwhelmingly positive [ $2,13,22,31,15,16,62]$.

Non-surgical treatments are described less commonly in the literature. Repeated nerve blocks have yielded positive outcomes $[11,19,23,24,59,60,62]$. Tricyclic antidepressants such as Gabapentin, which have been shown to reduce neurogenic pain, have been employed as part of a comprehensive treatment approach, along with other medications such as NSAIDS and methyl prednisone $[11,24,54,62]$. The efficacy of these interventions is not well established, as all reports of pharmacological management of IBSN injury were performed in conjunction with more invasive techniques, such as neurolysis, neurectomy, or nerve blocks.

Treatments involving only non-medical modalities are significantly less documented. As mentioned, only one account of physical therapy treatment by Pendergrass and Moore is found in the literature [18]. A 10-month program of lower extremity stretching and strengthening exercises with cryotherapy, compression, and activity modification for a 23-year-old patient who suffered a medial thigh trauma proved unsuccessful, and the patient ultimately underwent surgical debridement of scarring around the primary saphenous nerve.

Only a few other authors have described non-medical treatments. Dumitru and Windsor [11] described wrapping the knees of a 
competitive weightlifter who experienced a saphenous nerve injury secondary to a direct trauma to the medial knee. The patient responded well to treatment and was able to return to competitive weightlifting. No evidence was found that this treatment strategy has been replicated. In the chiropractic literature, Porr, Chrobak, and Muir [6] and Settergren [20] described successful conservative treatment of patients with suspected saphenous nerve injuries. Both reports included patients with non-surgical, traumatic injuries to the medial thigh and knee. Porr's, et al. treatment focused primarily on neuromusculoskeletal mobilization techniques, whereas Settergren described the utilization of Active Release Techniques ${ }^{\circledR}$ (ART) on the hip in an ultra-marathon runner. Neuromusculoskeletal mobilization has shown efficacy in the treatment of back and neck conditions, but its effect on other conditions remains unclear [65]. Little evidence supports the use of ART, with most reported evidence being anecdotal [66-69]. Consequently, the utility of these two approaches in treating patients with IBSN injury cannot be substantiated.

\section{Discussion and Implications for the Physical Therapist}

Injury to the saphenous nerve, particularly the infrapatellar branch, is a seemingly common condition, mostly associated with medial knee incisions during surgery and occasionally due to medial thigh or knee trauma. Fortunately, the majority of individuals who suffer injury experience relatively minor symptoms which are rarely disruptive to activities of daily living [3]. However, the literature contains a multitude of cases in which individuals experience debilitating pain, leading to loss of function. Such cases may be confounding for the clinician, as initial symptoms may be associated with postoperative pain or may mimic other conditions. The physical therapist, therefore, is tasked with recognizing recalcitrant hallmark signs and symptoms of IBSN injury. Should IBSN injury be suspected, a referral to an appropriate medical specialist for additional evaluation is appropriate.

Injury to the IBSN, specifically in postoperative cases where the patient may have suffered a nerve resection or entrapment, does not appear to respond well to physical therapy interventions $[12,24,62]$. In such cases, surgical intervention (neurolysis or neurectomy) or nerve blocks may be the most promising courses of treatment. In instances where superficial trauma to the thigh or knee describes the etiology, conservative treatment that addresses local inflammation, muscle imbalances, body mechanics, and tissue flexibility, may prove beneficial $[6,11,20]$. However, few reports of successful outcomes utilizing these interventions are documented, so the physical therapist should recognize the potential limitations of conservative approaches when treating this condition. Ultimately, the physical therapist must use the best clinical judgment in selecting a suitable course of action, and should make an appropriate referral, as indicated.

Financial Support: None

Competing Interests: The author declares no competing interests.

\section{Acknowledgements}

The author would like to thank Michael McKeough, PT, EdD for assistance with proofreading and editing.

\section{Abbreviations}

Infrapatellar branch of the saphenous nerve (IBSN); anterior cruciate ligament (ACL); total knee arthroplasty (TKA); reflex sympathetic dystrophy (RSD); deep vein thrombosis (DVT); magnetic resonance imaging (MRI); computerized tomography (CT); active release technique (ART)

\section{References}

1. Hosahalli, G., Sierakowski, A., Venkatramani. H., \& Sabapathy, S.R. (2017). Entrapment neuropathy of the infrapatellar branch of the saphenous nerve: treated by partial division of sartorius. Indian J Orthop, 51(4), 474-476.
2. Worth, R.M., Kettelkamp, D.B., Defalque, R.J., \& Duane, K.U. (1984). Saphenous nerve entrapment. The American Journal of Sports Medicine, 12(1), 80-81. https://doi. org/10.1177/036354658401200114

3. Figueroa, D., Calvo, R. Vaisman, A, Campero, M., \& Moraga, C. (2008). Injury to the infrapatellar branch of the saphenous nerve in ACL reconstruction with the hamstrings technique: clinical and electrophysiological study. Knee, (5), 360-3. doi: 10.1016/j.knee.2008.05.002.

4. Henry, B.M., Tomaszewski, K.A., Pękala, P.A., Graves, M.J., Pękala, Jr., Sanna, B., \& Mizia, E. (2018). Oblique incisions in hamstring tendon harvesting reduce iatrogenic injuries to the infrapatellar branch of the saphenous nerve. Knee Surgery, Sports Traumatology, Arthroscopy: Official Journal of the ESSKA, 26(4), 1197-1203. https://doi.org/10.1007/s00167-0174590-y

5. Mochida, H., \& Kikuchi, S. (1995. Injury to infrapatellar branch of saphenous nerve inarthroscopic knee surgery. Clin Orthop Relat Res. 320, 88-94.

6. Porr, J., Chrobak, K. \& Muir, B. (2013). Entrapment of the saphenous nerve at the adductor canal affecting the infrapatellar branch - a report on two cases. The Journal of the Canadian Chiropractic Association, 57(4), 341-349. Retrieved from https://www.ncbi.nlm.nih.gov/pmc/articles/PMC3845465/

7. Senegor, M. (1991). Iatrogenic saphenous neuralgia: successful therapy with neuroma resection. Neurosurgery, 28, 295-298.

8. Sherman, O.H., Fox, J.M., Del Pizzo, W., Friedman, M.J., Ferkel, R.D., \& Lawley, M.J. (1986). Arthroscopy "no problem surgery." An analysis of complications in two thousand six hundred and forty cases. J Bone Joint Surg Am, 68: 256-65.

9. Tifford, D.D., Spero, L., Luke, T., \& Plancher, K.D. (2000). The relationship of the infrapatellar branches of the saphenous nerve to arthroscopy portals and incisions for anterior cruciate ligament surgery. An anatomic study. Am J Sports Med, 28(4), 562-7. doi: 10.1177/03635465000280042001. PMID: 10921651.

10. Trescot, A.M., Brown, M.N., \& Karl, H.W. (2013). Infrapatellar saphenous neuralgia - diagnosis and treatment. Pain Physician, 16(3), E315-E324. https://doi.org/10.36076/ppj.2013/16/E315

11. Dumitru, D., \& Windsor, R.E. (1989). Subsartorial entrapment of thesaphenousnerve of a competitive weightlifter.Phys Sportsmed, 17(1),116-25. doi: 10.1080/00913847.1989.11709686. PMID: 27448006.

12. Hemler, D.E., Ward, WK, Karstetter, K.W., \& Bryant, P.M. (1991). Saphenous nerve entrapment caused by pes anserine bursitis mimicking stress fracture of the tibia. Archives of Physical Medicine and Rehabilitation, 72(5), 336-337.

13. House, J.H., \& Ahmed, K. (197). Entrapment neuropathy of the infrapatellar branch of the saphenous nerve. Am J Sports Med, 5(5), 217-24. doi: 10.1177/036354657700500509.

14. Kopell, H.P., \& Thompson, W.A. (1960). Knee pain due to saphenous nerve entrapment. $N$ Engl J Med, 263, 351-3.

15. Lippitt, A.B. (1993). Neuropathy of the saphenous nerve as a cause of knee pain. Bull Hosp Jt Dis, 52(2), 31-3.

16. Luerssen, T.G., Campbell, R.L., Defalque, R.J., \& Worth, R.M. (1983). Spontaneous saphenous neuralgia. Neurosurgery, 13(3), 238-41. doi: 10.1227/00006123-198309000-00004.

17. Peck, E., Finof, J.T., \& Smith, J. (2010). Neuropathies in runners. Clinics in Sports Medicine, 29(3), 437-457. https://doi. org/10.1016/j.csm.2010.03.002 
18. Pendergrass, T.L., \& Moore, J.H. (2004). Saphenous neuropathy following medial knee trauma. The Journal of Orthopaedic and Sports Physical Therapy, 34(6), 328-334.https://doi. org/10.2519/jospt.2004.1269

19. Romanoff, M.E., Cory, P.C., Kalenak, A., Keyser, G.C., \& adductor canal. The American Journal of Sports Medicine, 17(4), 478-481. https://doi.org/10.1177/036354658901700405

20. Settergren, R. (2012). Conservative management of a saphenous nerve entrapment in a female ultra-marathon runner. Journal of Bodywork and Movement Therapies, 17(3), 297-301. https:// doi.org/10.1016/j.jbmt.2012.10.005

21. Tennent, T.D., Birch, N.C., Holmes, M.J, Birch, R., \& Goddard, N.J. (1998). Knee pain and the infrapatellar branch of the saphenous nerve. J R Soc Med, 91(11), 573-575. doi:10.1177/014107689809101106

22. Ulloa, M., \& Coronel Banda, M. (2017). Scar tissue causing saphenous nerve entrapment: percutaneous scare release and fat grafting. Plastic and Reconstructive Surgery, 5(9), 1495. https:// doi.org/10.1097/GOX.0000000000001495

23. Clendenen, S., Greengrass, R., Whalen, J., \& O'Connor, M.I. (2015). Infrapatellar saphenous neuralgia after TKA can be improved with ultrasound-guided local treatments. Clin Orthop Relat Res, 473, 119-125.

24. Kalenak, A. (1996). Saphenous nerve entrapment. Operative Techniques in Sports Medicine, 4(1): 40-45. https://doi. org/10.1016/S1060-1872(96)80009-8

25. Ahadin T., Raissi, G.R., Togha, M., \& Nejati, P. (2020). Saphenous neuropathy in a patient with low back pain. Journal of brachial plexus and peripheral nerve injury, 5, 2. https://doi. org/10.1186/1749-7221-5-2

26. Mumenthaler, M., \& Schlaick, H. (1991). Peripheral Nerve Lesions, Diagnosis and Therapy. New York, NY: Thieme Medical Publishers

27. Papastergiou, S.G., Voulgaropoulos, H., Mikalef, P., Ziogas, E., Pappis, G., \& Giannakopoulos, I. (2006). Injuries to the infrapatellar branch(es) of the saphenous nerve in anterior cruciate ligament reconstruction with four-strand hamstring tendon autograft: vertical versus horizontal incision for harvest. Knee Surgery, Sports Traumatology, Arthroscopy: Official Journal of the ESSKA, 14(8), 789-793. https://doi.org/10.1007/ s00167-005-0008-3

28. Portland, G.H., Martin, D., Keene, G., \& Menz, T. (2005). Injury to the infrapatellar branch of the saphenous nerve in anterior cruciate ligament reconstruction: comparison of horizontal versus vertical harvest site incisions. Arthroscopy, 21(3), 281-5.

29. Abbott, L.C., \& Carpenter, W.F. (1945). Surgical approaches to the knee joint. Journal of Bone and Joint Surgery, 27, 277-310.

30. Kartus, J., Movin, T., \& Karlsson, J. (2001). Donor site morbidity and anterior knee problems after anterior cruciate ligament reconstruction using autografts. Current concepts. Arthroscopy, 17(9), 971-80.

31. Bertram, C., Porsch, M., Hackenbroch, M., \& Terhaag, D. (2000). Saphenous neuralgia after arthroscopically assisted anterior cruciate ligament reconstruction with a semitendinosus and gracilis tendon graft. Arthroscopy, 16 (7), 763-6

32. Kartus, J., Lindahl, S., Stener, S., Eriksson, B., \& Karlsson, J. (1999). Magneticc resonance imaging of the patellar tendon after harvesting its central third: a comparison between traditional and subcutaneous harvesting techniques. Arthroscopy, 15(6), 587-93. Marshall, W.K. (1989). Saphenous nerve entrapment at the

33. Kartus, J., Magnusson, L., Stener, S., Brandsson, S., Eriksson, B.I., \& Karlsson, J. (1999). Complications following arthroscopic anterior cruciate ligament reconstruction. A 2-5 year follow-up of 604 patients with special emphasis on anterior knee pain. Knee Surg Sports Traumatol Arthros, 7(1), 2-8.

34. Maeda, A., Shino, K., Horibe, S., \& Nakata, K., Buccafusca, G. (1996). Anterior cruciate ligament reconstruction with multistranded autogenous semitendinosus tendon. Am J Sports Med, 24, 504-9.

35. Sgaglione, N.A., Warren, R.F., Wickiewicz, T.L., Gold, D.A., \& Panariello, R.A. (1990) Primary repair with semitendinosus tendon augmentation of acute anterior cruciate ligament injuries. Am J Sports Med, 18, 64-73.

36. Hunter, L.Y., Louis, D.S., Ricciardi, J.R., \& O'Connor, G.A. (1979). The Saphenous nerve: its course and importance in medial arthrotomy. Am J Sports Med, 7(4), 227-30.

37. Lumsde, D.B, \& Kalenak, A. (1993). The saphenous nerve: an external method for identifying its exit from the adductor canal. Orthop Rev, 22(4), 451-5.

38. Patterson, D.C., Cirino, C.M., \& Gladstone, J.N. (2019). No safe zone: The anatomy of the saphenous nerve and its posteromedial branches. The Knee, 26(3), 660-665. https://doi.org/10.1016/j. knee.2019.02.010

39. Kartus, J., Ejerhed, L., \& Eriksson, B.I. (1999). The localization of the infrapatellar nerves in the anterior knee region with special emphasis on central third patellar tendon harvest: a dissection study on cadaver and amputated specimens. Arthroscopy, 15, 577-586

40. Arthornthurasook, A, \& Gaew-Im, K. (1988). Study of the infrapatellar nerve. American Journal of Sports Medicine, 16, 57-59. https://doi.org/10.1177/036354658801600110

41. Horner, G., \& Dellon, A.L. (1994). Innervation of the human knee joint and implications for surgery. Clin Orthop Relat Res, $301,221-226$.

42. Gali, J.C., Resina, A.F., Pedro, G.N., Ildefonso, A.M., Almagro, M.A.P., da Silva, P.A.C., \& Cateano, E.B. (2014). Importance of anatomically locating the infrapatellar branch of the saphenous nerve in reconstructing the anterior cruciate ligament using flexor tendons. Revista Brasileira de Ortopedia (English Ed.), 49(6), 625-629. https://doi.org/10.1016/j.rboe.2013.10.004

43. Sole, J.S., Pingree, M.J., Murthy, N.S., \& Sellon, J.L. (2014). Saphenous neuropathy secondary to extraneural ganglion cyst 15 years after reconstruction of the anterior cruciate ligament. Phys. Med. \& Rehab, 6(5), 451-455. doi: 10.1016/j. pmrj.2013.11.003.

44. Xiang, Y., Li, Z., Yu, P., Zheng, Z., Feng, B., \& Weng, X. (2019). Neuroma of the Infrapatellar branch of the saphenous nerve following total knee arthroplasty: a case report. $B M C$ Musculoskeletal Disorders, 20(1), 536-5. https://doi. org/10.1186/s12891-019-2934-0

45. Kachar, S.M., Williams, K.M., \& Finn, H.A. (2008). Neuroma of the infrapatellar branch of the saphenous nerve a cause of reversible knee stiffness after total knee arthroplasty. $J$ Arthroplasty, 23(6)n 927-30. doi: 10.1016/j.arth.2007.07.019. PMID: 18722295.

46. Ducic, I., Levin, M., Larson, E.E., \& Al-Attar, A. (2010). Management of chronic leg and knee pain following surgery or trauma related to saphenous nerve and knee neuromata. Ann Plast Surg, 64(1), 35-40.

47. Kummel, B.M., \& Zazanis, G.A. (1974). Preservation of infrapatellar branch of saphenous nerve during knee surgery. Orthopedics Review, 3(8), 43.

J Rehab Pract Res

Volume 2. 2021. 120 
48. Chambers, G.H. (1972). The prepatellar nerve: a cause of suboptimal results in knee arthrotomy. Clinical Orthhopedics, 82,157

49. Kerver, A.L., Leliveld, M.S., den Hartog, D., Verhofstad, M.H., \& Kleinrensink, G.J., (2013). The surgical anatomy of the infrapatellar branch of the saphenous nerve in relation to incisions for anteromedial knee surgery. J Bone Joint Surg Am, 95(23), 2119-25. doi: 10.2106/JBJS.L.01297.

50. Hakim, S.M., \& Narouze, S.N. (2015). Risk factors for chronic saphenous neuralgia following coronary artery bypass graft surgery utilizing saphenous vein grafts. Pain Practice, 15(8), 720-729. https://doi.org/10.1111/papr.12246

51. Mountney, J., \& Wilkinson, G.A. (1999). Saphenous neuralgia after coronary artery bypass grafting. Eur J Cardiothorac Surg, 16, 440-443.

52. Røder, O.C., Kamper, A., \& Jørgensen, S.J. (1984). Incidence of saphenous neuralgia in arterial surgery. Acta Chir Scand, $150(1), 23-4$.

53. Jones, N.A. (1978). Saphenous neuralgia: a complication of arterial surgery. Br J Surg, 65(7), 465-6. doi: 10.1002/ bjs. 1800650706.

54. Grabowski, R., Gobbi, A., Zabierek, S., \& Domzalski, M.E. (2018). Nonspecific chronic anteromedial knee pain neuroma as a cause of infrapatellar pain syndrome: Case study and literature review. Orthopaedic Journal of Sports Medicine, 6(1), 2325967117751042. https://doi. org $/ 10.1177 / 2325967117751042$

55. Harris, J.D., Fazalare, J.J., Griesser, M.J., \& Flanigan, D.C. (2012). Infrapatellar branch of saphenous neurectomy for painful neuroma: a case report. Am J Orthop, 41(1), 37-40.

56. Wijdicks, C.A., Westerhaus, B.D., Brand, E.J., Johansen, S., Engebresten, L., \& LaPrade R.F. (2010). Sartorial branch of the saphenous nerve in relation to a medial knee ligament repair or reconstruction. Knee Surgery, Sports Traumatology, Arthroscopy: Official Journal of the ESSKA, 18(8), 1105-1109. https://doi.org/10.1007/s00167-009-0934-6

57. Arct, W.A. (1968). Neuroma of the infrapatellar branch of the saphenous nerve and injury of saphenous nerve as causes of unexplained symptoms in the knee joint region. Wiad Lek (English Ed.), 21(11), 925-9.

58. Sahoo, R.K., Joshi, M., Joshi, S.D., \& Umamaheshwara, R.W. (2018). Treatment of bilateral refractory saphenous nerve entrapment neuropathy with pulsed radiofrequency. Anaesthesia, Pain \& Intensive Care, 22(1), 109.

59. Taheri, A., Hatami, M., Dashti, M., Khajehnasiri, A., \& Ghajarzadeh, M. (2015). Effect of ultrasound-guided subsartorial approach for saphenous nerve block in cases with saphenous nerve entrapment in adductor canal for controlling chronic knee pain. Middle East Journal of Anaesthesiology, 23(1), 25-28.
60. Pyne, D., Jawad, A.S.M., \& Padhiar, N. (2003). Saphenous nerve injury after fasciotomy for compartment syndrome. British Journal of Sports Medicine, 37(6), 541-542. https://doi. org/10.1136/bjsm.37.6.541

61. Tertemiz, O., Akcali, D., Köseoğlu, B.F., Ordu, G., Nilüfer, K., Ucar, M., ... \& Özçakar, L. Chronic unexplained thigh pain from saphenous nerve entrapment due to a leiomyoma. Pain Medicine, 16(2), 408-410. https://doi.org/10.1111/pme.12593

62. Mohile, N., Perez, J.R., Michael, E., Christopher, P., Foremny, G., Allegra, P., \& Greditzer, H.G. (2020). Chronic lower leg pain in athletes: Overview of presentation and management. HSS Journal, 16(1), 86-100. https://doi.org/10.1007/s11420019-09669-Z

63. Bademkiran, F., Obay, B., Aydogdu, I., \& Ertekin, C. (2007). Sensory conduction study of the infrapatellar branch of the saphenous nerve. Muscle \& Nerve, 35(2), 224-227. https://doi. org/10.1002/mus.20682

64. Locketz, A.J. (2004). Saphenous nerve conduction studies in suspected L4 radiculopathies: Friend or foe? Case report and literature review. Arch Phys Med Rehabil, 85(9), e23.

65. Basson, A., Olivier, B., Ellis, R., Coppieters, M., Stewart, A., \& Mudzi, W. (2017). The effectiveness of neural mobilization for neuromusculoskeletal conditions: A systematic review and meta-analysis. Journal of Orthopaedic \& Sports Physical Therapy, 47(9), 593-615.

66. Howitt, S., Jung, S., \& Hammonds, N. (2009). Conservative treatment of a tibialis posterior strain in a novice triathlete: A case report. The Journal of the Canadian Chiropractic Association, 53(1), 23-31.

67. George, T.W., Tepe, R.E., Busold, D., Keuss, S., Prather, H., $\&$ Skaggs, C.D. (2006). The effects of active release technique on carpal tunnel patients: A pilot study. Journal of chiropractic medicine, 119-122.

68. Howitt, S. (2006). Lateral epicondylosis: A case study of conservative care utilizing ART and rehabilitation. Journal of the Canadian Chiropractic Association, 50(3), 182-189.

69. Drover, J.M., Forand, D.R., \& Herzog, W. (2004). Influence of active release technique on quadriceps inhibition and strength: A pilot study. Journal of Manipulative and Physiological Therapeutics, 27(6), 408-413. 